\title{
Recent KLOE results on kaon branching ratios
}

\section{Patrizia de Simone on behalf of the KLOE/KLOE-2 collaborations* ${ }^{\dagger}$}

E-mail: patrizia.desimonedlnf.infn.it

The KLOE experiment at the Frascati $e^{+} e^{-}$collider DAФNE, operating at the $\phi$ peak, provides unique opportunities for precision measurements of kaon branching ratios. We present the upper limit on the branching ratio of the $\mathrm{CP}$ violating decay $\mathrm{K}_{\mathrm{S}} \rightarrow 3 \pi^{0}$ obtained with $1.7 \mathrm{fb}^{-1}$ of $e^{+} e^{-}$collisions collected by the KLOE detector. We also present the new preliminary result of the branching ratio for $\mathrm{K}^{+} \rightarrow \pi^{+} \pi^{-} \pi^{+}(\gamma)$ using approximately $10 \%$ of all KLOE data. This measurement supersedes the previous old result dating back to 1972 and completes the KLOE program of precise and fully inclusive measurements of the $\mathrm{K}^{ \pm}$dominant branching ratios.

2013 Kaon Physics International Conference,

29 April-1 May 2013

University of Michigan, Ann Arbor, Michigan - USA

*Speaker.

${ }^{\dagger}$ D. Babusci, D. Badoni, I. Balwierz-Pytko, G. Bencivenni, C. Bini, C. Bloise, F. Bossi, P. Branchini, A. Budano, L. Caldeira Balkest, G. Capon, F. Ceradini, P. Ciambrone, F. Curciarello, E. Czerwiński, E. Danè, V. De Leo, E. De Lucia, G. De Robertis, A. De Santis, P. de Simone, A. Di Domenico, C. Di Donato, R. Di Salvo, D. Domenici, O. Erriquez, G. Fanizzi, A. Fantini, G. Felici, S. Fiore, P. Franzini, A. Gajos, P. Gauzzi, G. Giardina, S. Giovannella, E. Graziani, F. Happacher, L. Heijkenskj, B. Höistad, L. Iafolla, M. Jacewicz, T. Johansson, K. Kacprzak, A. Kupsc, J. Lee-Franzini, B. Leverington, F. Loddo, S. Loffredo, G. Mandaglio, M. Martemianov, M. Martini, M. Mascolo, R. Messi, S. Miscetti, G. Morello, D. Moricciani, P. Moskal, F. Nguyen, A. Palladino, A. Passeri, V. Patera, I. Prado Longhi, A. Ranieri, C. F. Redmer, P. Santangelo, I. Sarra, M. Schioppa, B. Sciascia, M. Silarski, C. Taccini, L. Tortora, G. Venanzoni, W. Wiślicki, M. Wolke, J. Zdebik. 


\section{The KLOE experiment at DA $\Phi N E$}

DAФNE, the Frascati $\phi-$ factory [1], is an $e^{+} e^{-}$collider working at a center of mass energy of $\sqrt{s} \simeq m_{\phi} \simeq 1020 \mathrm{MeV}$. The $\phi$ production cross section is $\sim 3 \mu \mathrm{b}$. The beams collide at the interaction point (IP) with a crossing angle $\theta_{x} \simeq 25 \mathrm{mrad}$, therefore the $\phi$ 's are produced with a small momentum of $\sim 12.5 \mathrm{MeV}$ in the horizontal plane, and decay in almost collinear and monochromatic neutral (34\%) and charged (49\%) kaon pairs.

The KLOE detector consists of a large volume drift chamber surrounded by an electromagnetic calorimeter. A superconducting coil provides a $0.52 \mathrm{~T}$ solenoidal magnetic field. The tracking detector is a cylindrical drift chamber [2] (DC) $4 \mathrm{~m}$ diameter and $3.3 \mathrm{~m}$ long, with a total of $\sim 52000$ wires, of which $\sim 12000$ are sense wires. In order to minimize the multiple scattering and the $K_{L}$ regeneration, and to maximize the detection efficiency of low energy $\gamma$ 's, the DC works with an helium based gas mixture and its walls are made of light materials (mostly carbon fiber composites). The momentum resolution for tracks produced at large polar angle is $\sigma_{p \perp} / p_{\perp} \leq$ $0.4 \%$. Charged particle vertices are reconstructed with a spatial resolution of $\sim 3 \mathrm{~mm}[2]$. The fine sampling lead-scintillating fiber calorimeter [2] (EMC) consists of a barrel and two end-caps, and has solid angle coverage of $98 \%$. Photon energies and arrival times are measured with resolutions $\sigma_{E} / E=5.7 \% / \sqrt{E(\mathrm{GeV})}$ and $\sigma_{t}=57 \mathrm{ps} / \sqrt{E(\mathrm{GeV})} \oplus 100 \mathrm{ps}$, respectively. Photon entry points are determined with an accuracy $\sigma_{z} \sim 1 \mathrm{~cm} / \sqrt{E(\mathrm{GeV})}$ along the fibers and $\sigma_{\perp} \sim 1 \mathrm{~cm}$ in the transverse direction.

The unique feature of a $\phi-$ factory is the possibility of tagging. Specifically the detection of a $\mathrm{K}_{\mathrm{L}}\left(\mathrm{K}_{\mathrm{S}}\right)$ guarantees the presence of a $\mathrm{K}_{\mathrm{S}}\left(\mathrm{K}_{\mathrm{L}}\right)$ with known momentum and direction, the same holds for charged kaons pairs. The availability of tagged kaons enables the precision measurement of absolute BR's.

The $\mathrm{K}_{\mathrm{L}}$ beam is identified by the presence of a decay $\mathrm{K}_{\mathrm{S}} \rightarrow \pi^{+} \pi^{-}$. The $\mathrm{K}_{\mathrm{L}}$ momentum is given by the decay kinematics of $\phi \rightarrow \mathrm{K}_{\mathrm{L}} \mathrm{K}_{\mathrm{S}}$ using the reconstructed $\mathrm{K}_{\mathrm{S}}$ direction and the small velocity $v_{\phi}$ of the $\phi$ ( $v_{\phi}$ reconstructed run by run with the Bhabha events). The $\mathrm{K}_{\mathrm{S}}$ beam is identified by a called $\mathrm{K}_{\mathrm{L}}$-crash, i.e. an interaction of a $\mathrm{K}_{\mathrm{L}}$ in the EMC. A $\mathrm{K}_{\mathrm{L}}$-crash has a very clear signature consisting of a high energy deposit $(\mathrm{E}>100 \mathrm{MeV})$ in the calorimeter not associated to tracks and with an interaction time compatible with a velocity $\beta_{K_{L}} \simeq 0.22$. Furthermore the momentum and the direction of the $\mathrm{K}_{\mathrm{S}}$ is given by the kinematics of the $\phi$ decay.

The selection of the $\mathrm{K}^{ \pm}$beam is done reconstructing the 2 body decays $\mathrm{K}^{ \pm} \rightarrow \pi^{ \pm} \pi^{o}$ and $\mathrm{K}^{ \pm} \rightarrow \mu^{ \pm} \bar{v}(v)$, which are about $85 \%$ of the charged kaon decays. These decays are identified as two clear peaks in the momentum of the charged secondary tracks in the kaon rest frame.

KLOE completed the data taking in March 2006 with a total integrated luminosity of $\sim 2.5 \mathrm{fb}^{-1}$, corresponding to $\sim 7.5 \times 10^{9} \phi$-mesons produced.

\section{Direct search for $\mathrm{K}_{\mathrm{S}} \rightarrow \pi^{0} \pi^{0} \pi^{0}$}

The decay $\mathrm{K}_{\mathrm{S}} \rightarrow 3 \pi^{0}$ is a pure $\mathrm{CP}$ violating process. The related $\mathrm{CP}$ violation parameter $\eta_{000}$ is defined as the ratio of $\mathrm{K}_{\mathrm{S}}$ to $\mathrm{K}_{\mathrm{L}}$ decay amplitudes

$$
\eta_{000}=A\left(K_{S} \rightarrow 3 \pi^{0}\right) / A\left(K_{L} \rightarrow 3 \pi^{0}\right)=\varepsilon_{S}+\varepsilon_{000}^{\prime}
$$


where $\varepsilon_{S}$ quantify the $\mathrm{K}_{\mathrm{S}} \mathrm{CP}$ impurity and $\varepsilon_{000}^{\prime}$ is due to a direct $\mathrm{CP}$ violating term. In Chiral Pertubation Theory at the lowest order we have $\varepsilon_{000}^{\prime}=-2 \varepsilon^{\prime}$ [3] [4], where $\varepsilon^{\prime}$ is the direct CP violation parameter in $\pi \pi$ decays. Thus $\eta_{000} \simeq \varepsilon_{S}$, and therefore in the Standard Model one has (assuming CPT invariance, i.e. $\varepsilon_{S}=\varepsilon$ ) $\mathrm{BR}\left(\mathrm{K}_{\mathrm{S}} \rightarrow 3 \pi^{0}\right) \simeq 1.9 \times 10^{-9}$ to an accuracy of a few $\%$, making the direct observation of the decay a real challenge.

The best upper limit on BR( $\left.\mathrm{K}_{\mathrm{S}} \rightarrow 3 \pi^{0}\right)$ comes from the analysis of $450 \mathrm{pb}^{-1}$ data collected by the KLOE experiment [5]. Here we report the result of a new improved analysis using $1.7 \mathrm{fb}^{-1}$ of the KLOE data [6].

A $K_{L}$-crash tag and six neutral clusters coming from the IP are required in the search for $\mathrm{K}_{\mathrm{S}} \rightarrow \pi^{0} \pi^{0} \pi^{0}$. The background originates mainly from the $K_{S} \rightarrow 2 \pi^{0}$ events with two spurious clusters from fragmentation of the electromagnetic showers (so called splitting) or accidental activity. A smaller source of background comes from fake $\mathrm{K}_{\mathrm{L}}$ beam identification for $\phi \rightarrow K_{S} K_{L} \rightarrow$ $\pi^{+} \pi^{-}, 3 \pi^{0}$ events. In this events charged pions from $\mathrm{K}_{\mathrm{S}}$ decays interact in the DAФNE low$\beta$ intersection quadrupoles, ultimately simulating the $\mathrm{K}_{\mathrm{L}}$ interaction in the calorimeter, while $\mathrm{K}_{\mathrm{L}}$ decays close to the IP producing six $\gamma$ 's. To suppress this background we first reject events with charged particles coming from the vicinity of the IP, and then we cut on the reconstructed velocity and energy of the tagging $\mathrm{K}_{\mathrm{L}}$ mesons [7].

In the next stage of the analysis we perform a kinematic fit with 11 constraints: energy and momentum conservation, the kaon mass and the velocity of the six photons. In order to reject events with cluster splittings and accidentals we look at the correlations between two $\chi^{2}$-like discriminating variables: $\chi_{3 \pi}^{2}$ and $\chi_{2 \pi}^{2}$. The $\chi_{3 \pi}^{2}$ variable verifies the signal hypothesis by looking at the reconstructed masses of three pions. The $\chi_{2 \pi}^{2}$ variable is calculated selecting four out of the six clusters providing the best kinematic agreement with the $\mathrm{K}_{\mathrm{S}} \rightarrow \pi^{0} \pi^{0} \rightarrow 4 \gamma$ hypothesis. The pairing of the clusters is based on the requirement $m_{\gamma \gamma}=m_{\pi^{0}}$, and on the opening angle of the reconstructed pions trajectories in the $\mathrm{K}_{\mathrm{S}}$ center of mass frame. To improve the quality of the photon selection we cut on the variable

$$
\Delta E=M_{\phi} / 2-\sum E_{\gamma_{i}} / \sigma_{E}
$$

where the sum runs over the four $\gamma$ 's chosen to calculate $\chi_{2 \pi}^{2}$, and $\sigma_{E}$ is the appropriate energy resolution; for the signal events the missing $\pi^{0}$ implies $\Delta E \sim M_{\pi^{0}} / \sigma_{E}$. At the end of the analysis we cut also on the minimal distance between photon clusters, to refine the rejection of events with splitted clusters.

No events were found on data in the signal region, equally, no background events are found in the Montecarlo (MC) simulation (based on twice the data statistics), see fig 1. In the conservative assumption of no background we estimate an upper limit on the expected signal events of $U L\left(N_{3 \pi^{0}}\right)$ $=2.3$ at $90 \%$ C.L., with a signal selection efficiency $\varepsilon_{3 \pi^{0}}=0.23 \pm 0.01$.

In the same tagged sample we count $(1.142 \pm 0.005) \times 10^{8} \mathrm{~K}_{\mathrm{S}} \rightarrow \pi^{0} \pi^{0}$ events, used for signal normalization. Hence, we finally derive the upper limit

$$
\mathrm{BR}\left(\mathrm{K}_{\mathrm{S}} \rightarrow 3 \pi^{0}\right)<2.6 \times 10^{-8} \text { at } 90 \% \text { C.L. }
$$

which is almost five times lower than our previous result. This limit can be directly translated in: $\left|\eta_{000}\right|<0.0088$ at $90 \%$ C.L. 

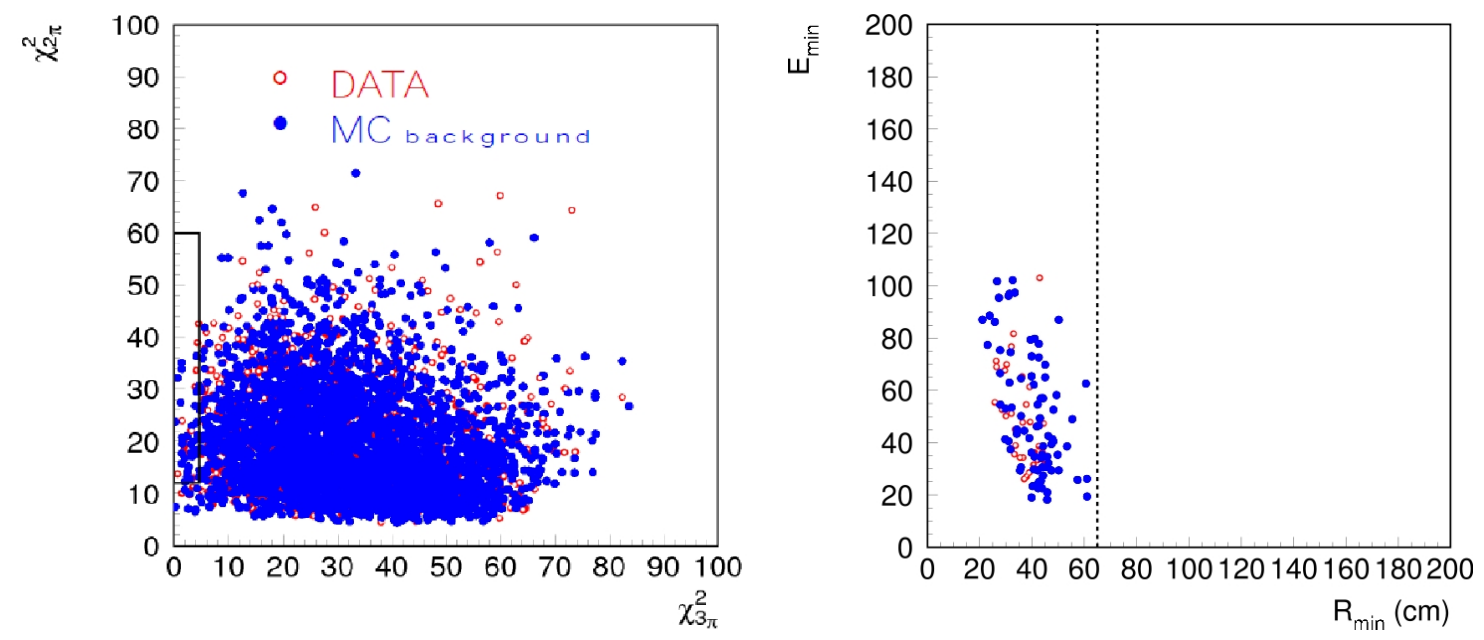

Figure 1: Left: $\chi_{2 \pi}^{2}$ versus $\chi_{3 \pi}^{2}$ for data (blue) and MC background (red); the rectangular regions represent the signal box. Right: distribution of the minimal energy of the cluster versus minimal distance $\left(\mathrm{R}_{\min }\right)$ between clusters in the events found in the signal box, for data (blue) and MC background (red); the dashed line correponds to the $\mathrm{R}_{\min }$ cut applied.

\section{Absolute $\mathrm{K}^{+} \rightarrow \pi^{+} \pi^{-} \pi^{+}(\gamma)$ branching ratio measurement}

The measurement of the BR of the $\mathrm{K}^{+} \rightarrow \pi^{+} \pi^{-} \pi^{+}(\gamma)$ decay completes the KLOE program of precise and fully inclusive kaon dominant BR's measurements. We have already published a value obtained fitting our $\mathrm{K}^{ \pm}$lifetime and BR's measurments [8], [9], [10], [11], [12], where the sum of the BR's is constrained to 1, [12]: $\mathrm{BR}\left(K^{ \pm} \rightarrow \pi^{ \pm} \pi^{+} \pi^{-}(\gamma)\right)=(5.68 \pm 0.22) \%$. Also the Flavianet working group gave a value using all the available measurements on charged kaons [13]: $\mathrm{BR}\left(K^{ \pm} \rightarrow\right.$ $\left.\pi^{ \pm} \pi^{+} \pi^{-}(\gamma)\right)=(5.73 \pm 0.16) \%$. The most recent result, $\mathrm{BR}\left(K^{ \pm} \rightarrow \pi^{ \pm} \pi^{+} \pi^{-}\right)=(5.56 \pm 0.20) \%$ [14], dates back to more than 30 years ago. Here we report about our new preliminary result obtained with $174 \mathrm{pb}^{-1}$ of the KLOE data.

To minimize the impact of the trigger efficiency on the signal side, we choose as normalization sample events given by the $K_{\mu 2}$ tags that provide by themself the calorimetric trigger of the event; this request reduces the normalization sample by $\sim 35 \%$.

To measure a branching ratio in KLOE, we must take in account a correction due to the bias on the tag selection due to the signal. We call this correction tag bias, and we evaluate it from the $\mathrm{MC}$ as the ratio of the MC branching ratio evaluated after the tag selection and the generated one. To measure the $\operatorname{BR}\left(K^{+} \rightarrow \pi^{+} \pi^{+} \pi^{-}(\gamma)\right)$, with the tag $K^{-} \rightarrow \mu^{-} v$ used as normalization sample, the tag bias correction that we must apply is: $C_{T B}=0.8386 \pm 0.0009$.

The track of the tagged kaon, $K^{-}$, is backward extrapolated to the interaction point, then the kinematic of the decay $\phi \rightarrow K^{+} K^{-}$, give us the possibility to define direction and momentum of the signal kaon, $K^{+}$. The decay products of the kaons have very low momentum, less than 200 $\mathrm{MeV} / \mathrm{c}$, and curl up in the KLOE magnetic field; this increase the probability to reconstruct broken tracks and fake vertices. We increase the quality of the reconstruction selecting events where the kaon decays before the inner wall of the DC $\left(\rho_{x y}=25 \mathrm{~cm}\right.$, geometrical acceptance $\left.\sim 26 \%\right)$ to 
reduce the maximum number of track inside the DC to three. Specifically we require at least two reconstructed tracks in the DC (pion candidates), then if their backward extrapolations do a vertex with the path of the signal kaon before the inner wall of the DC, we count the signal decays in the missing mass spectrum of the $3^{\text {th }}$ pion.

We select a vertex cutting on the distance of closest approach between each extrapolated tracks and the signal kaon path, DCA $<3 . \mathrm{cm}$, and on the distance of closest approach between the two selected tracks, $\mathrm{DCA}_{t t}<3$. $\mathrm{cm}$. To remove the background due to the 2-bodies decays, each selected track must have a momentum in the kaon rest frame less than $190 \mathrm{MeV} / \mathrm{c}$. After this selection, a residual background, mainly due to broken kaon tracks, is removed cutting tracks almost collinear, $\left|\cos \left(\theta_{12}\right)\right|<0.90$, where $\theta_{12}$ is the opening angle between the two selected tracks.
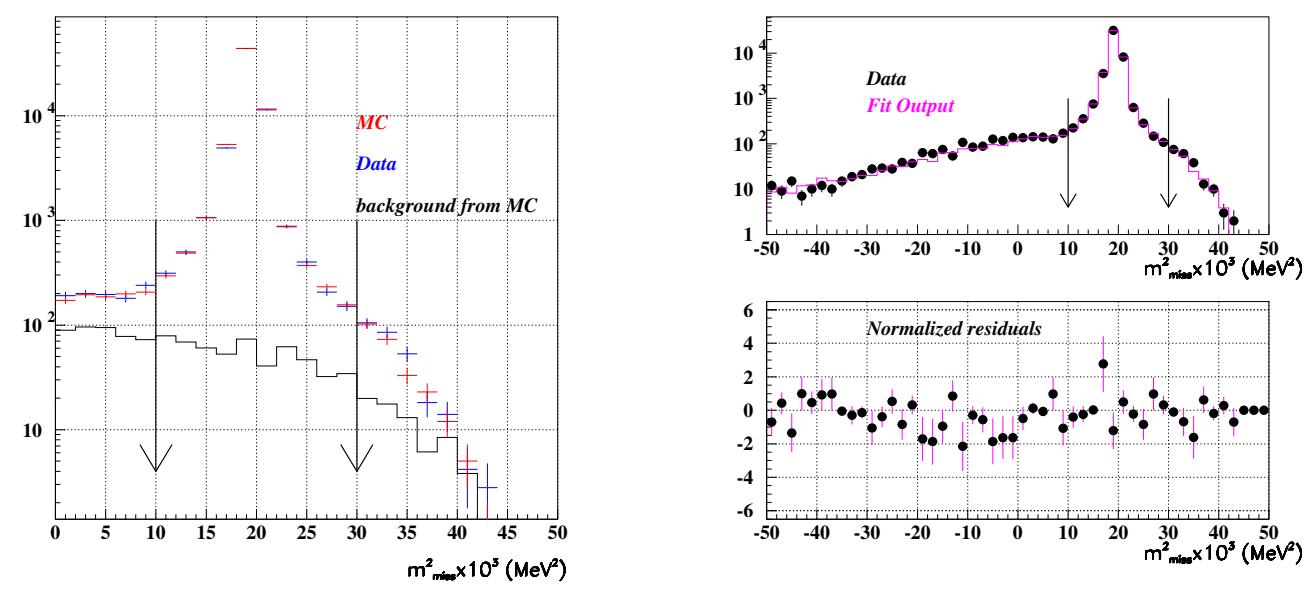

Figure 2: Left: missing mass spectrum; the data distribution is blue, the MC distribution is red, and in black is shown the MC background contribution. The lines correpond to the missing mass window. Right: fit of the missing mass spectrum (purple) superimposed with the data point (black). The bottom plot shows the normalized residuals of the fit.

The fig. 2 (left) shows the comparison between data (blue) and MC (red) of the missing mass spectrum, the MC background contribution (black) is superimposed; the signal over background ratio within the missing mass window $\left(10000\right.$. $<\mathrm{m}_{\text {miss }}^{2}<30000$.) $\mathrm{MeV}^{2}$ is $\simeq 88.40$. To extract the signal counts we fit the missing mass spectrum, where the shapes for the signal and background are given by the MC. The fig. 2 (right) shows the result of the fit (purple) superimposed with the data points (black), and the bottom plot shows the corresponding normalized residuals.

The selection efficiency is evaluated from MC, $\varepsilon_{\text {sel }}^{M C_{\text {true }}}$, and corrections are applied to account for data-MC differences in tracking. A control sample of positive kaons decaying in one negative pion plus whatever else, $K^{+} \rightarrow \pi^{-} X$, has been selected with a background contamination of $\simeq 10 \%$, to measure on data and MC the efficiency to reconstruct two tracks that satisfy the complete set of the signal selection cuts. The fig. 3 shows the selection efficiency for data (blue) and $\mathrm{MC}$ (red) as a function of the total transverse momentum $\mathrm{p}_{t} \mathrm{X}$ of the $\pi^{+} \pi^{+}$pair (left), and as a function of the total longitudinal momentum $\mathrm{p}_{l} \mathrm{X}$ of the $\pi^{+} \pi^{+}$pair (right). We measure the 

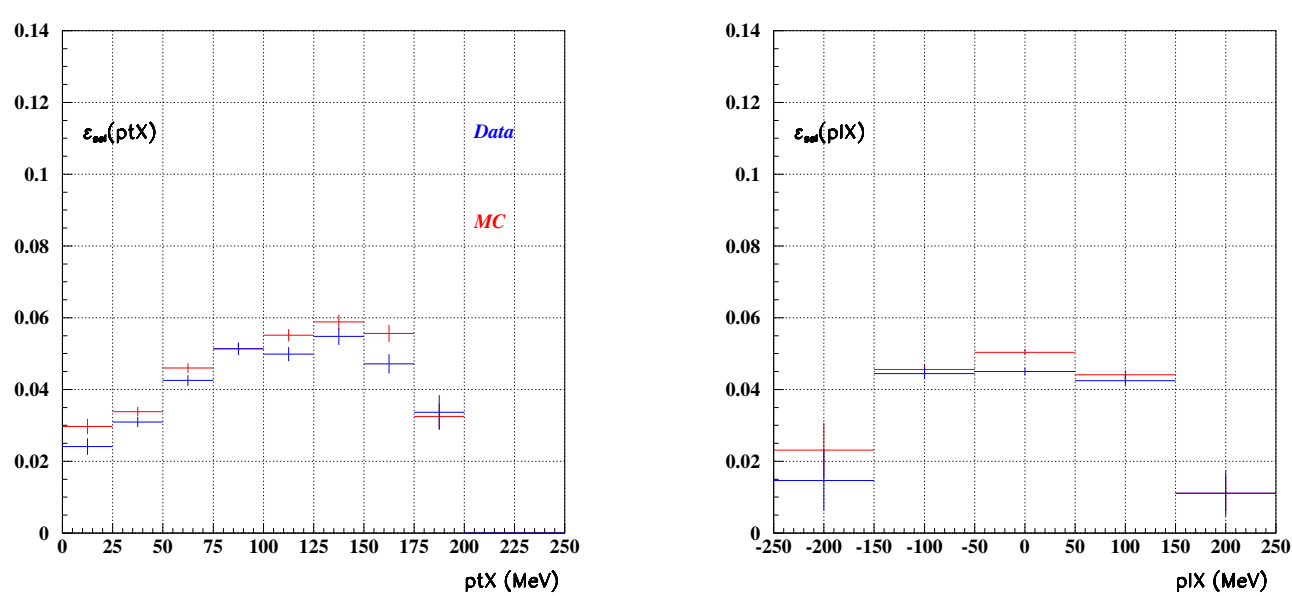

Figure 3: Left: selection efficiency for data (blue) and $\mathrm{MC}$ (red) as a function of the total transverse momentum $\mathrm{p}_{t} \mathrm{X}$ of the $\pi^{+} \pi^{+}$pair. Right: selection efficiency for data (blue) and MC (red) as a function of the total longitudinal momentum $\mathrm{p}_{l} \mathrm{X}$ of the $\pi^{+} \pi^{+}$pair.

corrections $\varepsilon_{\text {sel }}^{\text {data }} / \varepsilon_{\text {sel }}^{M C}$ as a function of $\mathrm{p}_{t} \mathrm{X}$ and $\mathrm{p}_{l} \mathrm{X}$, and the selection efficiency is obtained folding $\varepsilon_{\text {sel }}^{M C_{\text {true }}}$ with the corrections: $\varepsilon_{\text {sel }}=0.0793 \pm 0.0003$.

Finally we can extract our preliminary value:

$$
B R\left(K^{+} \rightarrow \pi^{+} \pi^{+} \pi^{-}(\gamma)\right)=0.05526 \pm 0.00035_{\text {stat }} \pm 0.00036_{\text {syst }}
$$

Main contributions to the systematic error are due to the cuts on the tracking variables, DCA and $\mathrm{DCA}_{t t}$.

\section{References}

[1] A. Drago, et al. LNF-03/012 (2003).

[2] F. Bossi, et al., Precision Kaon and Hadron Physics with KLOE, La Rivista del Nuovo Cimento, Vol.31, serie 5, N.10, (2008).

[3] L.F. Li and L. Wolffenstain, Phys. Rev. D 21, 178 (1908).

[4] L. Maiani, N. Paver $C P$ violation in $K \rightarrow 3 \pi$ decays, and G. D’Ambrosio, G. Isidori, A. Pugliese $C P$ and CPT measurements at DAФNE, in The second DAФNE physics handbook, Vol. 1, p.51-62 and 63-95, (1995)

[5] KLOE coll., F. Ambrosino, et al., Phys. Lett. 619, 61 (2005).

[6] KLOE-2 coll., D. Babusci, et al., Phys. Lett. B 723, 54 (2013).

[7] M. Silarski, Search for the CP symmetry violation in the decays of $\mathrm{K}_{\mathrm{S}}$ mesons using the KLOE detector, $\mathrm{PhD}$ thesis, Cracow (2012).

[8] KLOE coll., F. Ambrosino, et al., JHEP 01, 73 (2008).

[9] KLOE coll., F. Ambrosino, et al., Phys. Lett. B 632, 76 (2006). 
[10] KLOE coll., F. Ambrosino, et al., JHEP 02, 98 (2008).

[11] KLOE coll., A. Aloisio, et al., Phys. Lett. B 597, 139 (2004).

[12] KLOE coll., F.Ambrosino, et al., Phys. Lett. B 666, 15 (2008).

[13] M. Antonelli, et al., EPJC 69, 399 (2010).

[14] I.H. Chiang, PRD 6(1972), 1254. 